UDC 517.9

\title{
QUASILINEARIZATION FOR RESONANT BOUNDARY-VALUE PROBLEMS WITH MIXED BOUNDARY CONDITIONS ${ }^{*}$ \\ КВАЗІЛІНЕАРИЗАЦІЯ ДЛЯ ГРАНИЧНИХ ЗАДАЧ 3 РЕЗОНАНСОМ ТА МІІАНИМИ ГРАНИЧНИМИ УМОВАМИ
}

\author{
N. Sveikate \\ Daugavpils Univ. \\ Parades str., 1, Daugavpils, LV 5401, Latvia \\ e-mail: nsveikate@inbox.lv
}

\section{F. Sadyrbaev}

Univ. Latvia

Raina blvd., 19, Riga, LV 1586, Latvia

e-mail:felix@latnet.lv

We consider resonant problems of the type (i) $x^{\prime \prime}+p(t) x^{\prime}+q(t) x=f\left(t, x, x^{\prime}\right),(i i) x^{\prime}(0)=0, x(T)=0$, where $p, q, f$ are continuous functions and the homogeneous problem (iii) $x^{\prime \prime}+p(t) x^{\prime}+q(t) x=0$ with boundary-value conditions (ii) has a nontrivial solution. We study this problem by modifying the linear part and applying the quasilinearization technique to the modified problem.

Розглянуто задачі типу (i) $x^{\prime \prime}+p(t) x^{\prime}+q(t) x=f\left(t, x, x^{\prime}\right)$, (ii) $x^{\prime}(0)=0, x(T)=0$, з резонансом, де $p, q, f-$ неперервні функції та однорідна задача (iii) $x^{\prime \prime}+p(t) x^{\prime}+q(t) x=0$ разом з граничними умовами (ii) має нетривіальний розв'язок. Задача вивчається за допомогою зміни лінійної частини та застосування техніки квазілінеаризацї̈ до модифікованої задачі.

1. Introduction. It is well known that the two-point boundary-value problem (BVP)

$$
x^{\prime \prime}+p x^{\prime}+q x=F\left(t, x, x^{\prime}\right), \quad x^{\prime}(0)=0, \quad x(T)=0,
$$

where $p, q \in C(\mathbb{R})$ and $F \in C([0, T] \times R \times R, R)$ is a bounded function, has a $C^{2}$-solution if the linear part $\left(l_{2} x\right)(t):=x^{\prime \prime}+p x^{\prime}+q x$ is nonresonant with respect to the above boundary conditions. In other words, if the linear homogeneous problem

$$
x^{\prime \prime}+p x^{\prime}+q x=0, \quad x^{\prime}(0)=0, \quad x(T)=0,
$$

has only the trivial solution, then the problem (1.1) is solvable.

We consider the problem

$$
x^{\prime \prime}+p x^{\prime}+q x=f\left(t, x, x^{\prime}\right), \quad x^{\prime}(0)=0, \quad x(T)=0
$$

* This work has been supported by the European Social Fund within the Project "Support for the implementation of doctoral studies at Daugavpils University" Agreement Nr. 2009/0140/1DP/1.1.2.1.2/09/IPIA/VIAA/015.

(c) N. Sveikate, F. Sadyrbaev, 2014 
with a resonant linear part, where the right-hand side $f$ may be unbounded.

Problems of the type (1.2) arise when considering centrally symmetric solutions of elliptic partial differential equations. The center of symmetry (the origin) corresponds to the first boundary condition in (1.2).

The conditions for existence of a solution to the problem (1.2) are provided in the work [3].

To get existence and multiplicity results, we use the quasilinearization approach elaborated in the works $[6,7]$.

This method is based on the idea of replacing a given resonant problem with a nonresonant one and proving some estimates that ensure that there exists a bounded domain $D$ in the space of variables $\left(t, x, x^{\prime}\right)$ such that both the resonant and the nonresonant problems are equivalent in $D$.

The paper consists of the introduction, the definitions section, the section with Main Theorem and two sections where respectively nonresonant and resonant boundary-value problems are considered for a Emden - Fowler type equation.

\section{Definitions.}

Definition 2.1. We say that the linear part $\left(l_{2} x\right)(t)$ is $i$-nonresonant with respect to the mixed boundary conditions $x^{\prime}(0)=0, x(T)=0$ if a solution $x(t)$ of the Cauchy problem

$$
\left(l_{2} x\right)(t)=0, \quad x^{\prime}(0)=0, \quad x(0)=1
$$

has exactly $i$ zeros in the interval $(0, T)$ and $x(T) \neq 0$.

For instance, the linear expression $x^{\prime \prime}+10 x$ is 1-nonresonant in the interval $(0,1)$, since a solution of the respective Cauchy problem has exactly one zero in the interval.

Definition 2.2. We say that a solution $\xi(t)$ of the BVP (1.2) is of type $i, i=0,1, \ldots$, if the difference $x(t ; \alpha)-\xi(t)$ for sufficiently small $\alpha$ has exactly $i$ zeros in the interval $(0, T)$ and $x(T ; \alpha)-\xi(T) \neq 0$, where $x(t ; \alpha)$ is a solution of

$$
x^{\prime \prime}+p x^{\prime}+q x=f\left(t, x, x^{\prime}\right), \quad x^{\prime}(0)=\xi^{\prime}(0)=0, \quad x(0)=\xi(0)+\alpha .
$$

Remark 2.1. Definition 2.2 fits for the case of $f\left(t, x, x^{\prime}\right)$ in (2.1) being merely continuous. If $f$ has continuous partial derivatives $f_{x}$ and $f_{x^{\prime}}$, the equation of variations

$$
y^{\prime \prime}+p(t) y^{\prime}+q(t) y=f_{x}\left(t, \xi(t), \xi^{\prime}(t)\right) y+f_{x^{\prime}}\left(t, \xi(t), \xi^{\prime}(t)\right) y^{\prime}, \quad y^{\prime}(0)=0, \quad y(0)=1,
$$

can be considered and the definition then becomes as follows: a solution $\xi(t)$ is of type $i$ if $y(t)$ in (2.2) has exactly $i$ zeros in $(0, T)$ and $y(T) \neq 0$.

3. Main theorem. Consider the quasilinear problem (1.1), where the right-hand side $F$ is bounded.

To the end of this section we assume that the linear part in (1.1) is nonresonant.

Lemma 3.1. If the continuous function $F$ in (1.1) is bounded, then problem (1.1) has a solution.

This is a well-known result that follows from Conti's theorem [2]. 
Lemma 3.2. $A$ set $S$ of all solutions of problem (1.1) is compact in $C^{1}([0, T])$.

This can be proved by a routine application of the Arzela-Ascoli criterion using the Green's function representation of a solution to the BVP.

Corollary 3.1. A set of initial data $\{x(0)\}$ of all solutions to the BVP (1.1) is compact in $R$.

Proof. Follows from compactness of the set $S$ of all solutions of problem (1.1).

Consider solutions $x(t ; \gamma)$ of the Cauchy problem

$$
x^{\prime \prime}+p x^{\prime}+q x=F\left(t, x, x^{\prime}\right), \quad x^{\prime}(0)=0, \quad x(0)=\gamma .
$$

Lemma 3.3. Let $\xi(t)$ be any solution of problem (1.1), where the linear part $\left(l_{2} x\right)(t)$ is $i$ nonresonant.

Then for $\alpha$ large enough the difference $u(t, \alpha)=x(t ; \alpha)-\xi(t)$ has exactly $i$ zeros in $(0, T)$ and $u(T ; \alpha) \neq 0$.

Essentially Lemma 2.3 in [6].

The next result on separation of zeros is of Valle Poussin type [5] (Chapter 3, Section 17).

Lemma 3.4. Let $\xi(t)$ be any solution of problem (1.1), where the linear part $\left(l_{2} x\right)(t)$ is nonresonant, and $u(t ; \alpha)$ is as in Lemma 3.3.

Zeros $t_{i}(\alpha)$ (if any) of the function $u(t ; \alpha)$ continuously depend on $\alpha$. If $|\alpha|<B<+\infty$, then there exists $\delta(B)>0$ such that the distance between two consecutive zeros of $u(t ; \alpha)$ cannot be less that $\delta$. If $u\left(T ; \alpha_{0}\right)=0$ for some $\alpha_{0} \neq \xi(0)$ then the respective $x\left(t ; \alpha_{0}\right)$ solves problem (1.1).

Essentially Lemma 2.4 in [6].

Theorem 3.1. A quasilinear problem (1.1) with $i$-nonresonant linear part has a solution $x(t)$ of type $i$.

Proof. Consider a set $S$ of all solutions of problem (1.1). This set is compact in $C^{1}([0, T])$ and a set of the initial conditions $x(0)$ of elements of $S$ is compact in $R$. Therefore there exist solutions $x_{\max }(t)$ and $x_{\min }(t)$ with the maximal and minimal values of $x(0)$ respectively. In case of uniqueness of a solution these solutions coincide.

Consider a solution $x_{\max }(t)$ with the property that $x_{\max }(0)=\max _{S}\{x(0)\}$. We claim that this solution possesses the property of being a solution of type $i$. Suppose this is not the case. Consider solutions $x(t ; \gamma)$ of the Cauchy problem for $\gamma>x_{\max }(0)$. For $\gamma$ tending to $+\infty$ the functions $u(t ; \gamma)=x(t ; \gamma)-x_{\max }(t)$ have exactly $i$ zeros and $u(T ; \gamma) \neq 0$, by Lemma 3.3.

Consider the following cases.

Case 1. There exists a sequence $\left\{\gamma_{n}\right\}, \gamma_{n}>x_{\max }(0)$, such that the differences $x\left(t ; \gamma_{n}\right)-$ $-x_{\max }(t)$ have more than $i$ zeros in $(0, T]$. If $x\left(T ; \gamma_{n}\right)-x_{\max }(T)=0$ for some $n$, then a solution $x\left(t ; \gamma_{n}\right)$ solves problem (1.1). This is in contradiction with the assumption that $x_{\max }(t)$ is a solution of problem (1.1) with the maximal initial value $x(0)$. Therefore we may suppose that the differences $x\left(t ; \gamma_{n}\right)-x_{\max }(t)$ have more than $i$ zeros in the open interval $(0, T)$. Denote $t_{i+1}\left(\gamma_{n}\right)$ the $(i+1)$-st zero of the differences $x\left(t ; \gamma_{n}\right)-x_{\max }(t)$. By Lemma 3.4, it is separated from neighboring zeros $t_{i}\left(\gamma_{n}\right)$ and, if any, from $t_{i+2}\left(\gamma_{n}\right)$. Moreover, $t_{i+1}(\gamma)$ changes continuously together with $\gamma=x(0)$. Thus there exists $\gamma_{*}>x_{\max }(0)$ such that the respective solution $x\left(t ; \gamma_{*}\right)$ has its $(i+1)$-st zero at $t=T$. Therefore this solution of the equation solves also the 
BVP (1.1). The contradiction with the maximality property of $x_{\max }(t)$ is obtained. Therefore the considered case is not possible.

Case 2. There exists a sequence $\left\{\gamma_{n}\right\}, \gamma_{n}>x_{\max }(0)$, such that the differences $x\left(t ; \gamma_{n}\right)-$ $-x_{\max }(t)$ have less than $i$ zeros in $(0, T]$. If $x\left(T ; \gamma_{n}\right)-x_{\max }(T)=0$ for some $n$, then a solution $x\left(t ; \gamma_{n}\right)$ solves the problem (1.1). The contradiction follows as in the above case. Suppose that the differences $x\left(t ; \gamma_{n}\right)-x_{\max }(t)$ have less than $i$ zeros in the open interval $(0, T)$. Denote by $t_{i-1}\left(\gamma_{n}\right)$ the $(i-1)$-th zero (if $\left.i \geq 1\right)$ of the differences $x\left(t ; \gamma_{n}\right)-x_{\max }(t)$. It is separated from neighboring zeros if $\gamma \in\left(x_{\max }(0), \Gamma\right)$, where $\Gamma$ is a large positive number such that a solution $x(t ; \Gamma)$ of the Cauchy problem (3.1) (where $\gamma=\Gamma$ ) has exactly $i$ zeros in $(0, T)$ and does not vanish at $t=T$. An extra zero $t_{i}(\gamma)$ has to appear in the interval $(0, T]$ then. Suppose a solution $x\left(t ; \gamma^{*}\right)$ is such a solution. Then it solves problem (1.1) and, therefore, a contradiction is obtained with the maximality property of $x_{\max }(t)$.

If $i=0$, the consideration may be conducted similarly.

Theorem 3.1 is proved.

4. Multiple solutions. The results of the previous section allow for treating multiple solutions of the BVP (1.2). Indeed, suppose that the problem can be reformulated so that the right-hand side is bounded and the modified problem hence has a solution. If the graph $\left(t, x(t), x^{\prime}(t)\right)$ of this solution lies in the domain $D_{i}$ where the original and the modified equations are equivalent, then $x(t)$ solves also the original problem. Hence the existence. Moreover, if the linear part of the modified problem is $i$-nonresonant then there exists a solution $x(t)$ of the same type $i$.

Suppose that this process can be repeated with some other linear part of type $j \neq i$. Then it can be concluded that the original problem also has a solution of type $j$. Hence the second conditions.

In the sequel we will show that this approach is possible for both the nonresonant and even the resonant the problems.

To be specific, we choose to treat the Emden - Fowler type equations.

5. Mixed problem. Nonresonant case. Consider the problem

$$
\begin{gathered}
x^{\prime \prime}+r^{2} x=-Q(t)|x|^{p} \operatorname{sign} x, \\
x^{\prime}(0)=0, \quad x(1)=0,
\end{gathered}
$$

where the linear part $x^{\prime \prime}+r^{2} x$ is nonresonant with respect to the boundary conditions, that is $r \neq \frac{\pi}{2}+\pi n, n=0,1,2, \ldots$

We show that it is possible to quasilinearize equation in (5.1) so that multiple solutions are revealed in the spirit of Main Theorem 3.1.

Theorem 5.1. Suppose that

$$
0<q_{1} \leq Q(t) \leq q_{2}
$$

and the inequality

$$
\frac{k^{2}}{\sqrt{k^{2}+r^{2}} \cos \sqrt{k^{2}+r^{2}}}<\beta \frac{p^{\frac{p}{p-1}}}{|p-1|}\left(\frac{q_{1}}{q_{2}}\right)^{\frac{1}{|p-1|}}
$$




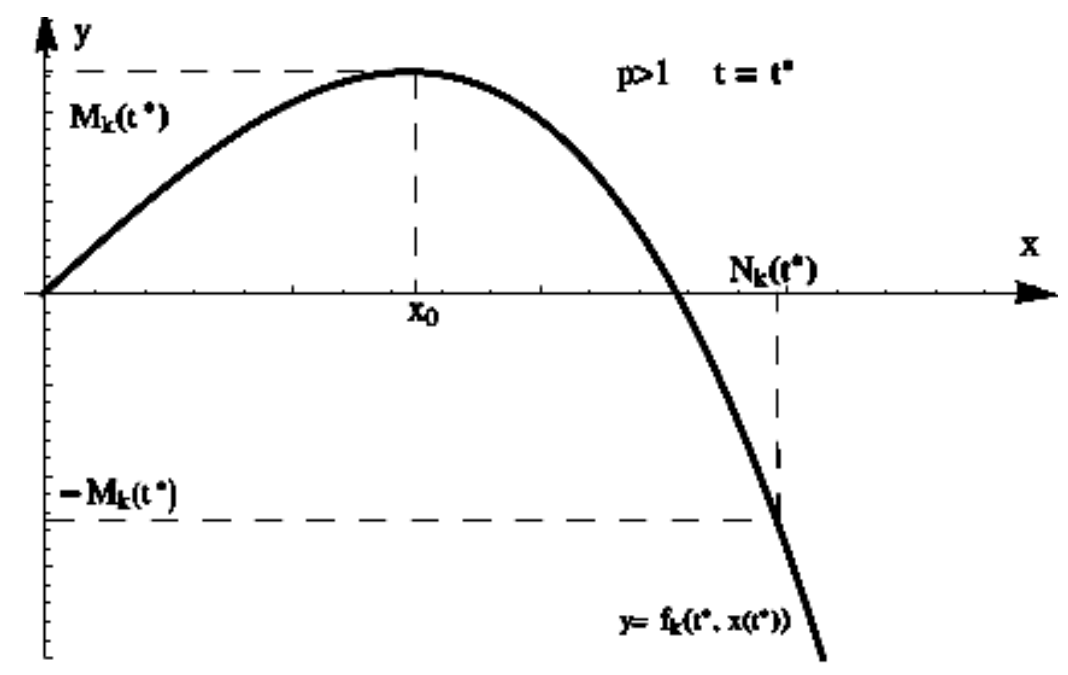

Fig 5.1. Existence of a number $N_{k}$.

holds for some $k^{2} \in\left(\frac{\pi^{2}(2 i-1)^{2}}{4}-r^{2}, \frac{\pi^{2}(2 i+1)^{2}}{4}-r^{2}\right), i=1,2, \ldots$, where $\beta>1$ is a root of the equation

$$
\beta^{p}=\beta+(p-1) p^{\frac{p}{1-p}}
$$

Then there exists an i-type solution of problem (5.1), (5.2).

Proof. We use the quasilinearization approach.

First we modify equation (5.1) adding a linear part

$$
x^{\prime \prime}+r^{2} x+k^{2} x=k^{2} x-Q(t)|x|^{p} \operatorname{sign} x .
$$

The linear part $\left(L_{2} x\right)(t):=x^{\prime \prime}+\left(r^{2}+k^{2}\right) x$ is nonresonant with respect to the boundary conditions (5.2).

We define

$$
f_{k}(t, x):=k^{2} x-Q(t)|x|^{p} \operatorname{sign} x
$$

We wish to bound the right-hand side in (5.6). Since it is odd in $x$ for fixed $t$, let us consider it for nonnegative values of $x$. An extremum of $f_{k}(t, x)$ is attained (maximum for $p>1$ and minimum for $0<p<1$ ) at the point

$$
x_{0}=\left(\frac{k^{2}}{p Q(t)}\right)^{\frac{1}{p-1}} .
$$

We can calculate the value of the function at the point of maximum $x_{0}$, see Fig. 5.1. Set

$$
M_{k}(t)=\left|f_{k}\left(t, x_{0}\right)\right|=\left(\frac{k^{2}}{p}\right)^{\frac{p}{p-1}}|p-1| Q^{\frac{1}{1-p}}
$$


We choose constants $N_{k}$ so that

$$
\forall(t, x): t \in[0,1], \quad|x| \leq N_{k} \Rightarrow\left|f_{k}(t, x)\right| \leq M_{k}
$$

The value of $N_{k}(t)$ can be computed by solving the equation

$$
f_{k}(t, x)=-f_{k}\left(t, x_{0}\right),
$$

or, equivalently, that of

$$
k^{2} x-Q(t) x^{p}=\left(\frac{k^{2}}{p}\right)^{\frac{p}{p-1}}|p-1| Q^{\frac{1}{1-p}}
$$

with respect to $x$ for any fixed $t$. Computation gives that

$$
N_{k}(t)=\left(\frac{k^{2}}{Q(t)}\right)^{\frac{1}{p-1}} \beta,
$$

where the constant $\beta$ is a positive root of the equation (5.5).

Then let us consider the quasilinear equation

$$
x^{\prime \prime}+\left(r^{2}+k^{2}\right) x=F_{k}(t, x),
$$

where $F_{k}(t, x)=f_{k}\left(\delta\left(-N_{k}, x, N_{k}\right)\right)$ and $\delta$ is a "cut-of" function, that is,

$$
\delta\left(-N_{k}, x, N_{k}\right)= \begin{cases}N_{k}, & x>N_{k}, \\ x, & -N_{k} \leq x \leq N_{k}, \\ -N_{k}, & x<N_{k}\end{cases}
$$

and $\max \left\{\left|F_{k}\right|: x \in R\right\}=M_{k}$.

The original problem (5.1), (5.2) and the quasilinear one (5.7), (5.2) are equivalent in the domain $\Omega_{k}=\left\{(t, x): 0 \leq t \leq 1,|x(t)| \leq N_{k}\right\}$. Problem (5.7), (5.2) can be written in the integral form,

$$
x_{k}(t)=\int_{0}^{1} G_{k}(t, s) F_{k}(s, x(s)) d s,
$$

where $G_{k}(t, s)$ is Green's function $[1,4]$ to the respective homogeneous problem

$$
x^{\prime \prime}+\left(r^{2}+k^{2}\right) x=0, \quad x^{\prime}(0)=x(1)=0 .
$$

It is given by

$$
G_{k}(t, s)= \begin{cases}\frac{\sin \sqrt{r^{2}+k^{2}}(s-1) \cos \sqrt{r^{2}+k^{2}} t}{\sqrt{r^{2}+k^{2}} \cos \sqrt{r^{2}+k^{2}}}, & 0 \leq t \leq s \leq 1, \\ \frac{\sin \sqrt{r^{2}+k^{2}}(t-1) \cos \sqrt{r^{2}+k^{2}} s}{\sqrt{r^{2}+k^{2}} \cos \sqrt{r^{2}+k^{2}}}, & 0 \leq s<t \leq 1,\end{cases}
$$

ISSN 1562-3076. Нелінійні коливання, 2014, m. 17, № 1 
and satisfies the estimate

$$
\left|G_{k}(t, s)\right| \leq \Gamma_{k}=\frac{1}{\sqrt{r^{2}+k^{2}}\left|\cos \sqrt{r^{2}+k^{2}}\right|}
$$

It follows from (5.8) that

$$
|x(t)| \leq \Gamma_{k} M_{k}
$$

If the inequality

$$
\Gamma_{k} M_{k}<N_{k}
$$

holds, then a solution $x(t)$ of the quasilinear problem (5.7), (5.2) satisfies the estimate

$$
|x(t)|<N_{k} \quad \forall t \in[0,1]
$$

and the original problem (5.1), (5.2) allows for quasilinearization with respect to the domain $\Omega_{k}$ and the linear part $\left(L_{2} x\right)(t):=x^{\prime \prime}+\left(r^{2}+k^{2}\right) x$. It follows from Main Theorem 3.1 that if the linear part $\left(L_{2} x\right)(t)$ is $i$-nonresonant, then problem (5.1), (5.2) has an $i$-type solution.

Consider inequality (5.9) and assume that $Q(t)$ satisfies estimates (5.3). If $p>1$, then

$$
\begin{gathered}
\max _{t \in[0,1]} M_{k}(t)=\left(\frac{k^{2}}{p}\right)^{\frac{p}{p-1}}|p-1| q_{1}^{\frac{1}{1-p}}, \\
\min _{t \in[0,1]} N_{k}(t)=\left(\frac{k^{2}}{q_{2}}\right)^{\frac{1}{p-1}} \beta ;
\end{gathered}
$$

but in the case of $0<p<1$ we have

$$
\begin{gathered}
\max _{t \in[0,1]} M_{k}(t)=\left(\frac{k^{2}}{p}\right)^{\frac{p}{p-1}}|p-1| q_{2}^{\frac{1}{1-p}}, \\
\min _{t \in[0,1]} N_{k}(t)=\left(\frac{k^{2}}{q_{1}}\right)^{\frac{1}{p-1}} \beta .
\end{gathered}
$$

Hence inequality (5.9) reduces to (5.4).

Theorem 5.1 proved.

Corollary 5.1. If there exist numbers $k^{2} \in\left(\frac{\pi^{2}(2 i-1)^{2}}{4}-r^{2}, \frac{\pi^{2}(2 i+1)^{2}}{4}-r^{2}\right), i=1,2, \ldots$ $\ldots, n$, which satisfy inequality (5.4), then there exist at least $n$ solutions of different types to problem (5.1), (5.2). 
Table 5.1. Results of calculations for the nonresonant BVP $x^{\prime \prime}+\pi^{2} x=-|x|^{p} \operatorname{sign} x, x^{\prime}(0)=0=x(1)$, $p>1$

\begin{tabular}{|c|c|c|c|c|c|}
\hline$p$ & $i$ & Nonresonant intervals & $k$ & $\Gamma_{k} M_{k}$ & $N_{k}$ \\
\hline 3 & 1 & $\left(-\frac{\pi \sqrt{3}}{2} ; \frac{\pi \sqrt{5}}{2}\right)$ & $\frac{\pi}{2}$ & 0.456 & 1.814 \\
\hline \multirow[t]{2}{*}{2} & 1 & $\left(-\frac{\pi \sqrt{3}}{2} ; \frac{\pi \sqrt{5}}{2}\right)$ & $\frac{\pi}{2}$ & 0.465 & 2.978 \\
\hline & 2 & $\left(\frac{\pi \sqrt{5}}{2} ; \frac{\pi \sqrt{21}}{2}\right)$ & $\frac{3 \pi}{2}$ & 26.738 & 26.806 \\
\hline \multirow[t]{3}{*}{$\frac{5}{4}$} & 1 & $\left(-\frac{\pi \sqrt{3}}{2} ; \frac{\pi \sqrt{5}}{2}\right)$ & $\frac{\pi}{2}$ & 2.289 & 47.492 \\
\hline & 2 & $\left(\frac{\pi \sqrt{5}}{2} ; \frac{\pi \sqrt{21}}{2}\right)$ & $\frac{3 \pi}{2}$ & 95945.8 & 311593.0 \\
\hline & 3 & $\left(\frac{\pi \sqrt{21}}{2} ; \frac{\pi \sqrt{45}}{2}\right)$ & $\frac{5 \pi}{2}$ & $1.521 \cdot 10^{7}$ & $1.855 \cdot 10^{7}$ \\
\hline \multirow[t]{3}{*}{$\frac{6}{5}$} & 1 & $\left(-\frac{\pi \sqrt{3}}{2} ; \frac{\pi \sqrt{5}}{2}\right)$ & $\frac{\pi}{2}$ & 4.617 & 117.828 \\
\hline & 2 & $\left(\frac{\pi \sqrt{5}}{2} ; \frac{\pi \sqrt{21}}{2}\right)$ & $\frac{3 \pi}{2}$ & $1.742 \cdot 10^{6}$ & $6.958 \cdot 10^{6}$ \\
\hline & 3 & $\left(\frac{\pi \sqrt{21}}{2} ; \frac{\pi \sqrt{45}}{2}\right)$ & $\frac{5 \pi}{2}$ & $7.669 \cdot 10^{8}$ & $11.507 \cdot 10^{8}$ \\
\hline \multirow[t]{4}{*}{$\frac{8}{7}$} & 1 & $\left(-\frac{\pi \sqrt{3}}{2} ; \frac{\pi \sqrt{5}}{2}\right)$ & $\frac{\pi}{2}$ & 20.599 & 722.124 \\
\hline & 2 & $\left(\frac{\pi \sqrt{5}}{2} ; \frac{\pi \sqrt{21}}{2}\right)$ & $\frac{3 \pi}{2}$ & $6.296 \cdot 10^{8}$ & $34.539 \cdot 10^{8}$ \\
\hline & 3 & $\left(\frac{\pi \sqrt{21}}{2} ; \frac{\pi \sqrt{45}}{2}\right)$ & $\frac{5 \pi}{2}$ & $2.139 \cdot 10^{12}$ & $14.408 \cdot 10^{12}$ \\
\hline & 4 & $\left(\frac{\pi \sqrt{45}}{2} ; \frac{\pi \sqrt{77}}{2}\right)$ & $\frac{7 \pi}{2}$ & $4.601 \cdot 10^{14}$ & $4.898 \cdot 10^{14}$ \\
\hline \multirow[t]{6}{*}{$\frac{21}{20}$} & 1 & $\left(-\frac{\pi \sqrt{3}}{2} ; \frac{\pi \sqrt{5}}{2}\right)$ & $\frac{\pi}{2}$ & $9.462 \cdot 10^{5}$ & $917.88 \cdot 10^{5}$ \\
\hline & 2 & $\left(\frac{\pi \sqrt{5}}{2} ; \frac{\pi \sqrt{21}}{2}\right)$ & $\frac{3 \pi}{2}$ & $7.351 \cdot 10^{25}$ & $111.594 \cdot 10^{25}$ \\
\hline & 3 & $\left(\frac{\pi \sqrt{21}}{2} ; \frac{\pi \sqrt{45}}{2}\right)$ & $\frac{5 \pi}{2}$ & $1.464 \cdot 10^{35}$ & $8.348 \cdot 10^{35}$ \\
\hline & 4 & $\left(\frac{\pi \sqrt{45}}{2} ; \frac{\pi \sqrt{77}}{2}\right)$ & $\frac{7 \pi}{2}$ & $1.984 \cdot 10^{41}$ & $5.844 \cdot 10^{41}$ \\
\hline & 5 & $\left(\frac{\pi \sqrt{77}}{2} ; \frac{\pi \sqrt{117}}{2}\right)$ & $\frac{9 \pi}{2}$ & $7.575 \cdot 10^{45}$ & $13.567 \cdot 10^{45}$ \\
\hline & 6 & $\left(\frac{\pi \sqrt{117}}{2} ; \frac{\pi \sqrt{165}}{2}\right)$ & $\frac{11 \pi}{2}$ & $3.456 \cdot 10^{49}$ & $4.154 \cdot 10^{49}$ \\
\hline
\end{tabular}

For instance, we consider the BVP

$$
x^{\prime \prime}+\pi^{2} x=-|x|^{p} \operatorname{sign} x, \quad x^{\prime}(0)=0, \quad x(1)=0 .
$$

The linear part $\left(L_{2} x\right)(t):=x^{\prime \prime}+\pi^{2} x$ is nonresonant with respect to the given boundary conditions. To modify the differential equation we use values of $k$ in the form $\frac{\pi}{2}+\pi n, n=0,1,2, \ldots$ We obtained that for some values of $p$ inequality (5.4) holds. The obtained results are given in Tables 5.1 and 5.2. 
Table 5.2. Results of calculations for the nonresonant BVP $x^{\prime \prime}+\pi^{2} x=-|x|^{p} \operatorname{sign} x, \quad x^{\prime}(0)=0=$ $=x(1), 0<p<1$

\begin{tabular}{|c|c|c|c|c|c|}
\hline$p$ & $i$ & Nonresonant intervals & $k$ & $\Gamma_{k} M_{k}$ & $N_{k}$ \\
\hline \multirow[t]{6}{*}{$\frac{20}{21}$} & 1 & $\left(-\frac{\pi \sqrt{3}}{2} ; \frac{\pi \sqrt{5}}{2}\right)$ & $\frac{\pi}{2}$ & $7.837 \cdot 10^{-4}$ & $770.654 \cdot 10^{-4}$ \\
\hline & 2 & $\left(\frac{\pi \sqrt{5}}{2} ; \frac{\pi \sqrt{21}}{2}\right)$ & $\frac{3 \pi}{2}$ & $7.577 \cdot 10^{-30}$ & $70.431 \cdot 10^{-30}$ \\
\hline & 3 & $\left(\frac{\pi \sqrt{21}}{2} ; \frac{\pi \sqrt{45}}{2}\right)$ & $\frac{5 \pi}{2}$ & $5.863 \cdot 10^{-39}$ & $33.894 \cdot 10^{-39}$ \\
\hline & 4 & $\left(\frac{\pi \sqrt{45}}{2} ; \frac{\pi \sqrt{77}}{2}\right)$ & $\frac{7 \pi}{2}$ & $8.273 \cdot 10^{-45}$ & $24.703 \cdot 10^{-45}$ \\
\hline & 5 & $\left(\frac{\pi \sqrt{77}}{2} ; \frac{\pi \sqrt{117}}{2}\right)$ & $\frac{9 \pi}{2}$ & $3.545 \cdot 10^{-49}$ & $6.437 \cdot 10^{-49}$ \\
\hline & 6 & $\left(\frac{\pi \sqrt{117}}{2} ; \frac{\pi \sqrt{165}}{2}\right)$ & $\frac{11 \pi}{2}$ & $1.155 \cdot 10^{-52}$ & $1.407 \cdot 10^{-52}$ \\
\hline \multirow[t]{4}{*}{$\frac{7}{8}$} & 1 & $\left(-\frac{\pi \sqrt{3}}{2} ; \frac{\pi \sqrt{5}}{2}\right)$ & $\frac{\pi}{2}$ & $2.693 \cdot 10^{-5}$ & $97.983 \cdot 10^{-5}$ \\
\hline & 2 & $\left(\frac{\pi \sqrt{5}}{2} ; \frac{\pi \sqrt{21}}{2}\right)$ & $\frac{3 \pi}{2}$ & $3.998 \cdot 10^{-12}$ & $22.762 \cdot 10^{-12}$ \\
\hline & 3 & $\left(\frac{\pi \sqrt{21}}{2} ; \frac{\pi \sqrt{45}}{2}\right)$ & $\frac{5 \pi}{2}$ & $3.002 \cdot 10^{-15}$ & $6.421 \cdot 10^{-15}$ \\
\hline & 4 & $\left(\frac{\pi \sqrt{45}}{2} ; \frac{\pi \sqrt{77}}{2}\right)$ & $\frac{7 \pi}{2}$ & $2.669 \cdot 10^{-17}$ & $2.948 \cdot 10^{-17}$ \\
\hline \multirow[t]{3}{*}{$\frac{5}{6}$} & 1 & $\left(-\frac{\pi \sqrt{3}}{2} ; \frac{\pi \sqrt{5}}{2}\right)$ & $\frac{\pi}{2}$ & $2.237 \cdot 10^{-4}$ & $149.065 \cdot 10^{-4}$ \\
\hline & 2 & $\left(\frac{\pi \sqrt{5}}{2} ; \frac{\pi \sqrt{21}}{2}\right)$ & $\frac{3 \pi}{2}$ & $2.690 \cdot 10^{-9}$ & $11.302 \cdot 10^{-9}$ \\
\hline & 3 & $\left(\frac{\pi \sqrt{21}}{2} ; \frac{\pi \sqrt{45}}{2}\right)$ & $\frac{5 \pi}{2}$ & $1.559 \cdot 10^{-11}$ & $2.460 \cdot 10^{-11}$ \\
\hline \multirow[t]{3}{*}{$\frac{4}{5}$} & 1 & $\left(-\frac{\pi \sqrt{3}}{2} ; \frac{\pi \sqrt{5}}{2}\right)$ & $\frac{\pi}{2}$ & $6.751 \cdot 10^{-4}$ & $149.065 \cdot 10^{-4}$ \\
\hline & 2 & $\left(\frac{\pi \sqrt{5}}{2} ; \frac{\pi \sqrt{21}}{2}\right)$ & $\frac{3 \pi}{2}$ & $7.306 \cdot 10^{-8}$ & $25.244 \cdot 10^{-8}$ \\
\hline & 3 & $\left(\frac{\pi \sqrt{21}}{2} ; \frac{\pi \sqrt{45}}{2}\right)$ & $\frac{5 \pi}{2}$ & $1.176 \cdot 10^{-9}$ & $11.526 \cdot 10^{-9}$ \\
\hline \multirow[t]{2}{*}{$\frac{1}{2}$} & 1 & $\left(-\frac{\pi \sqrt{3}}{2} ; \frac{\pi \sqrt{5}}{2}\right)$ & $\frac{\pi}{2}$ & 0.031 & 0.239 \\
\hline & 2 & $\left(\frac{\pi \sqrt{5}}{2} ; \frac{\pi \sqrt{21}}{2}\right)$ & $\frac{3 \pi}{2}$ & 0.002 & 0.003 \\
\hline$\frac{1}{3}$ & 1 & $\left(-\frac{\pi \sqrt{3}}{2} ; \frac{\pi \sqrt{5}}{2}\right)$ & $\frac{\pi}{2}$ & 0.075 & 0.397 \\
\hline
\end{tabular}

For instance, if $p=\frac{5}{4}$, there exist at least three values $k_{0}=\frac{\pi}{2}, k_{1}=\frac{3 \pi}{2}$ and $k_{2}=\frac{5 \pi}{2}$, which satisfy inequality (5.4). For example,

$$
x^{\prime \prime}+\pi^{2} x=-|x|^{\frac{5}{4}} \operatorname{sign} x, \quad x^{\prime}(0)=0, \quad x(1)=0,
$$

has solutions of different types (see Fig. 5.2-5.4). 


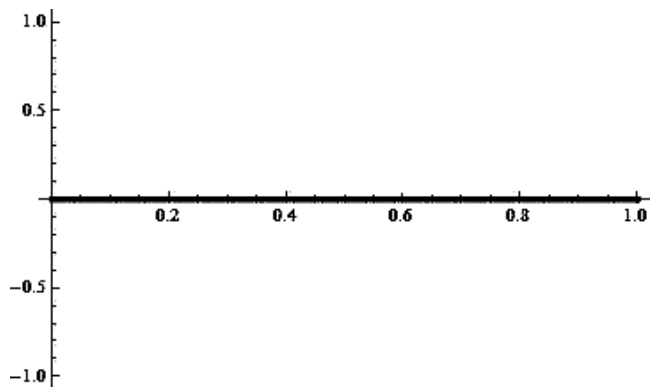

(a)

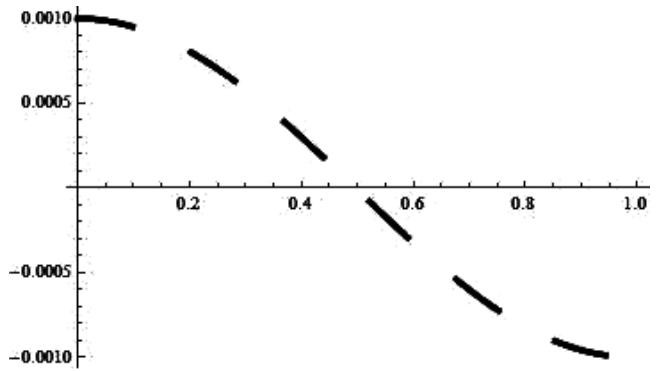

(b)

Fig. 5.2. 1-Type solution of the problem (5.10): $(a)$ the trivial solution of problem $(5.10),(b)$ the difference between the neighboring solution and the trivial solution of problem (5.10).

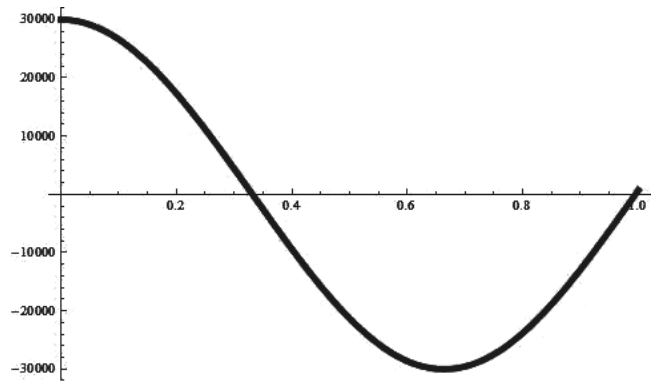

(a)

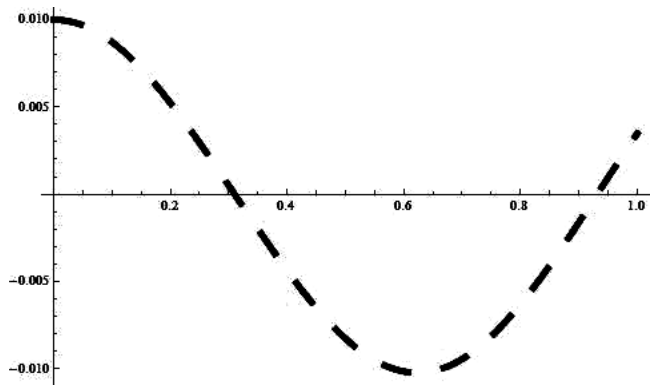

(b)

Fig. 5.3. 2-Type solution of problem (5.10): (a) a solution of problem (5.10) given for the initial data $\varepsilon_{2}^{\prime}(0)=0, \varepsilon_{2}(0)=30000,(b)$ the difference between the neighboring solution and the solution $\varepsilon_{2}(t)$ of problem (5.10).

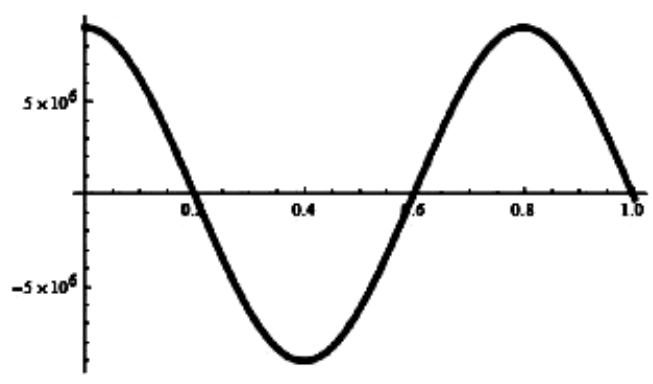

(a)

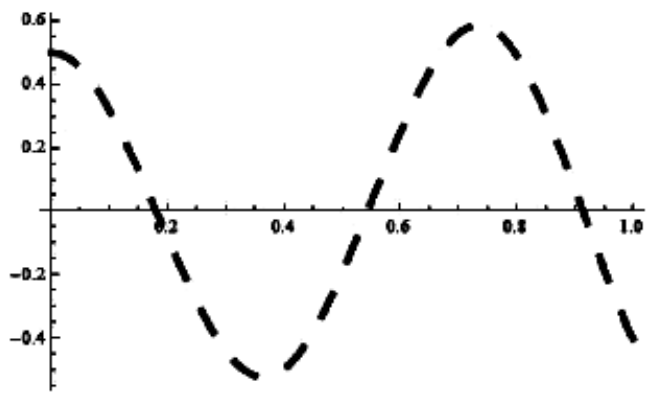

(b)

Fig. 5.4. 3-Type solution of the problem (5.10): (a) a solution of problem (5.10) given for the initial data $\varepsilon_{3}^{\prime}(0)=0, \varepsilon_{3}(0)=9000000,(b)$ the difference between the neighboring solution and the solution $\varepsilon_{3}(t)$ of problem $(5.10)$.

6. Mixed problem. Resonant case. Consider the problem

$$
x^{\prime \prime}+\left(\frac{\pi}{2}\right)^{2} x=-Q(t)|x|^{p} \operatorname{sign} x,
$$




$$
x^{\prime}(0)=0, \quad x(1)=0
$$

where the linear part $x^{\prime \prime}+k^{2} x$ is resonant with respect to the boundary conditions, since the linear Cauchy problem

$$
x^{\prime \prime}+\left(\frac{\pi}{2}\right)^{2} x=0, \quad x^{\prime}(0)=0, \quad x(0)=1,
$$

has the nontrivial solution $x(t)=\cos \frac{\pi}{2} t$.

We show that it is possible to quasilinearize equation in (6.1) so that multiple solutions are revealed in the spirit of Main Theorem 3.1.

Theorem 6.1. Suppose that

$$
0<q_{1} \leq Q(t) \leq q_{2}
$$

and the inequality

$$
\frac{2 k^{2}}{\sqrt{\pi^{2}+4 k^{2}} \cos \frac{\sqrt{\pi^{2}+4 k^{2}}}{2}}<\beta \frac{p^{\frac{p}{p-1}}}{|p-1|}\left(\frac{q_{1}}{q_{2}}\right)^{\frac{1}{|p-1|}}
$$

holds for some $k \in(\pi \sqrt{i(i-1)}, \pi \sqrt{i(i+1)}), i=1,2, \ldots$, where $\beta>1$ is a root of the equation

$$
\beta^{p}=\beta+(p-1) p^{\frac{p}{1-p}} .
$$

Then there exists an i-type solution of problem (6.1), (6.2).

Proof. Now we modify equation (6.1) adding a linear part so that the resulting linear part becomes nonresonant.

The proof is conducted as that of Theorem 5.1, replacing $r^{2}$ with $\left(\frac{\pi}{2}\right)^{2}$ in all formulas.

Corollary 6.1. If there exist numbers $k \in(\pi \sqrt{i(i-1)}, \pi \sqrt{i(i+1)}), i=1,2, \ldots, n$, which satisfy inequality (6.3), then there exist at least $n$ solutions of different types to problem (6.1), (6.2).

We obtained the results (see Table 6.1) for certain values of $p$, which show that some numbers $k$ in the form $\frac{\pi}{2}+\pi n, n=0,1,2, \ldots$, for any respective nonresonance interval satisfy inequality (6.1). 
Table 6.1. Results of calculations for the resonant BVP $x^{\prime \prime}+\frac{\pi^{2}}{4} x=-|x|^{p} \operatorname{sign} x, \quad x^{\prime}(0)=0=x(1)$

\begin{tabular}{|c|c|c|c|c|c|}
\hline$p$ & $i$ & Nonresonant intervals & $k$ & $\Gamma_{k} M_{k}$ & $N_{k}$ \\
\hline 3 & 1 & $(0 ; \pi \sqrt{2})$ & $\frac{\pi}{2}$ & 1.348 & 1.814 \\
\hline 2 & 1 & $(0 ; \pi \sqrt{2})$ & $\frac{\pi}{2}$ & 1.131 & 2.978 \\
\hline$\frac{5}{4}$ & 1 & $(0 ; \pi \sqrt{2})$ & $\frac{\pi}{2}$ & 5.568 & 47.492 \\
\hline \multirow[t]{2}{*}{$\frac{6}{5}$} & 1 & $(0 ; \pi \sqrt{2})$ & $\frac{\pi}{2}$ & 11.233 & 117.828 \\
\hline & 2 & $(\pi \sqrt{2} ; \pi \sqrt{6})$ & $\frac{3 \pi}{2}$ & $6.413 \cdot 10^{6}$ & $6.958 \cdot 10^{6}$ \\
\hline \multirow[t]{2}{*}{$\frac{8}{7}$} & 1 & $(0 ; \pi \sqrt{2})$ & $\frac{\pi}{2}$ & 50.118 & 722.124 \\
\hline & 2 & $(\pi \sqrt{2} ; \pi \sqrt{6})$ & $\frac{3 \pi}{2}$ & $2.318 \cdot 10^{9}$ & $3.454 \cdot 10^{9}$ \\
\hline \multirow[t]{3}{*}{$\frac{21}{20}$} & 1 & $(0 ; \pi \sqrt{2})$ & $\frac{\pi}{2}$ & $2.302 \cdot 10^{6}$ & $91.789 \cdot 10^{6}$ \\
\hline & 2 & $(\pi \sqrt{2} ; \pi \sqrt{6})$ & $\frac{3 \pi}{2}$ & $2.706 \cdot 10^{26}$ & $11.159 \cdot 10^{26}$ \\
\hline & 3 & $(\pi \sqrt{6} ; \pi \sqrt{12})$ & $\frac{5 \pi}{2}$ & $5.676 \cdot 10^{35}$ & $8.348 \cdot 10^{35}$ \\
\hline \multirow[t]{4}{*}{$\frac{41}{40}$} & 1 & $(0 ; \pi \sqrt{2})$ & $\frac{\pi}{2}$ & $8.151 \cdot 10^{13}$ & $644.211 \cdot 10^{13}$ \\
\hline & 2 & $(\pi \sqrt{2} ; \pi \sqrt{6})$ & $\frac{3 \pi}{2}$ & $1.165 \cdot 10^{53}$ & $9.522 \cdot 10^{53}$ \\
\hline & 3 & $(\pi \sqrt{6} ; \pi \sqrt{12})$ & $\frac{5 \pi}{2}$ & $1.829 \cdot 10^{71}$ & $5.329 \cdot 10^{71}$ \\
\hline & 4 & $(\pi \sqrt{12} ; \pi \sqrt{20})$ & $\frac{7 \pi}{2}$ & $1.761 \cdot 10^{83}$ & $2.611 \cdot 10^{83}$ \\
\hline \multirow[t]{4}{*}{$\frac{40}{41}$} & 1 & $(0 ; \pi \sqrt{2})$ & $\frac{\pi}{2}$ & $1.379 \cdot 10^{-18}$ & $109.800 \cdot 10^{-18}$ \\
\hline & 2 & $(\pi \sqrt{2} ; \pi \sqrt{6})$ & $\frac{3 \pi}{2}$ & $1.003 \cdot 10^{-56}$ & $8.254 \cdot 10^{-56}$ \\
\hline & 3 & $(\pi \sqrt{6} ; \pi \sqrt{12})$ & $\frac{5 \pi}{2}$ & $1.809 \cdot 10^{-74}$ & $5.309 \cdot 10^{-74}$ \\
\hline & 4 & $(\pi \sqrt{12} ; \pi \sqrt{20})$ & $\frac{7 \pi}{2}$ & $3.701 \cdot 10^{-86}$ & $5.528 \cdot 10^{-86}$ \\
\hline \multirow[t]{3}{*}{$\frac{20}{21}$} & 1 & $(0 ; \pi \sqrt{2})$ & $\frac{\pi}{2}$ & $1.907 \cdot 10^{-10}$ & $77.065 \cdot 10^{-10}$ \\
\hline & 2 & $(\pi \sqrt{2} ; \pi \sqrt{6})$ & $\frac{3 \pi}{2}$ & $1.645 \cdot 10^{-29}$ & $7.043 \cdot 10^{-29}$ \\
\hline & 3 & $(\pi \sqrt{6} ; \pi \sqrt{12})$ & $\frac{5 \pi}{2}$ & $2.274 \cdot 10^{-38}$ & $3.389 \cdot 10^{-38}$ \\
\hline \multirow[t]{2}{*}{$\frac{7}{8}$} & 1 & $(0 ; \pi \sqrt{2})$ & $\frac{\pi}{2}$ & $6.552 \cdot 10^{-5}$ & $97.983 \cdot 10^{-5}$ \\
\hline & 2 & $(\pi \sqrt{2} ; \pi \sqrt{6})$ & $\frac{3 \pi}{2}$ & $1.472 \cdot 10^{-11}$ & $2.276 \cdot 10^{-11}$ \\
\hline \multirow[t]{2}{*}{$\frac{6}{7}$} & 0 & $(0 ; \pi \sqrt{2})$ & $\frac{\pi}{2}$ & $1.866 \cdot 10^{-4}$ & $24.247 \cdot 10^{-4}$ \\
\hline & 1 & $(\pi \sqrt{2} ; \pi \sqrt{6})$ & $\frac{3 \pi}{2}$ & $3.772 \cdot 10^{-10}$ & $5.069 \cdot 10^{-10}$ \\
\hline \multirow[t]{2}{*}{$\frac{5}{6}$} & 1 & $(0 ; \pi \sqrt{2})$ & $\frac{\pi}{2}$ & 0.001 & 0.006 \\
\hline & 2 & $(\pi \sqrt{2} ; \pi \sqrt{6})$ & $\frac{3 \pi}{2}$ & $9.903 \cdot 10^{-9}$ & $11.302 \cdot 10^{-9}$ \\
\hline$\frac{4}{5}$ & 1 & $(0 ; \pi \sqrt{2})$ & $\frac{\pi}{2}$ & 0.002 & 0.015 \\
\hline$\frac{3}{4}$ & 1 & $(0 ; \pi \sqrt{2})$ & $\frac{\pi}{2}$ & 0.005 & 0.037 \\
\hline
\end{tabular}

ISSN 1562-3076. Нелінійні коливання, 2014, m. 17, № 1 


\begin{tabular}{cccccc}
\hline$p$ & $i$ & Nonresonant intervals & $k$ & $\Gamma_{k} M_{k}$ & $N_{k}$ \\
\hline$\frac{2}{3}$ & 1 & $(0 ; \pi \sqrt{2})$ & $\frac{\pi}{2}$ & 0.018 & 0.093 \\
\hline$\frac{1}{2}$ & 1 & $(0 ; \pi \sqrt{2})$ & $\frac{\pi}{2}$ & 0.075 & 0.239 \\
\hline
\end{tabular}

Example. Consider the BVP

$$
x^{\prime \prime}+\left(\frac{\pi}{2}\right)^{2} x=-|x|^{\frac{6}{5}} \operatorname{sign} x, \quad x^{\prime}(0)=x(1)=0 .
$$

The function $-|x|^{\frac{6}{5}} \operatorname{sign} x$ is odd. And for $x>0$ we can rewrite

$$
x^{\prime \prime}+\left(\frac{\pi}{2}\right)^{2} x=-x^{\frac{6}{5}} .
$$

Rewrite (6.5) equivalently

$$
x^{\prime \prime}+\left(\frac{\pi}{2}\right)^{2} x+\left(\frac{\pi}{2}\right)^{2} x=\left(\frac{\pi}{2}\right)^{2} x-x^{\frac{6}{5}} .
$$

The linear part in (6.6) is no more resonant with respect to (6.2).

We would like to make the function $f(x):=\frac{\pi^{2}}{4} x-x^{\frac{6}{5}}$ bounded and still continuous. The function $f(x)$ is an odd function with a maximum at $x=\frac{3125}{7962624} \pi^{10}$. Define $M:=$ $:=f\left(\frac{3125}{7962624} \pi^{10}\right)=\frac{3125}{191102976} \pi^{12}$. Solve the equation $f(x)=-M$ for $x>0$. The solution is $N:=117.828$.

Define the truncated function

$$
F(x)= \begin{cases}-\frac{3125}{191102976} \pi^{12}, & x>117,828 \\ \frac{\pi^{2}}{4} x-x^{\frac{6}{5}}, & -117,828 \leq x \leq 117,828 \\ \frac{3125}{191102976} \pi^{12}, & x<-117.828\end{cases}
$$

The function $F(x)$ is continuous and bounded by the number $M$. Therefore the quasilinear problem

$$
x^{\prime \prime}+\frac{\pi^{2}}{2} x=F(x), \quad x^{\prime}(0)=0, \quad x(1)=0,
$$

has a solution $x(t)$. Let us show that $|x(t)| \leq N \forall t \in[0,1]$ and hence $x(t)$ is also a solution of problem (6.1), (6.2). A solution $x(t)$ satisfies the integral equation

$$
x(t)=\int_{0}^{1} G(t, s) F(x(s)) d s,
$$




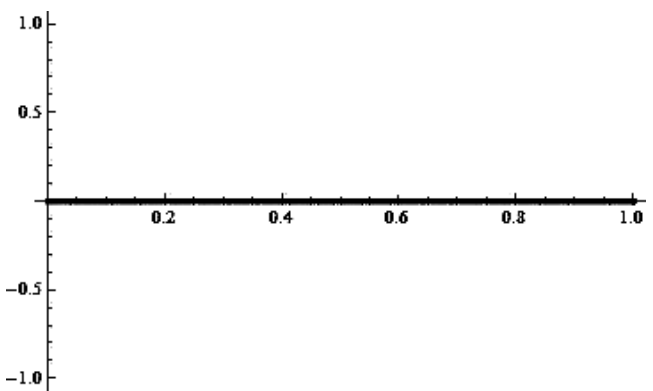

(a)

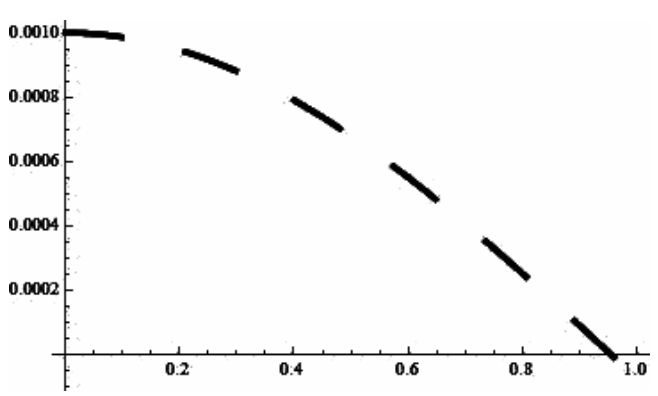

(b)

Fig. 6.1. 1-Type solution of the problem (6.4): (a) the trivial solution of problem (6.4), (b) the difference between the neighboring solution and the trivial solution of problem (6.4).

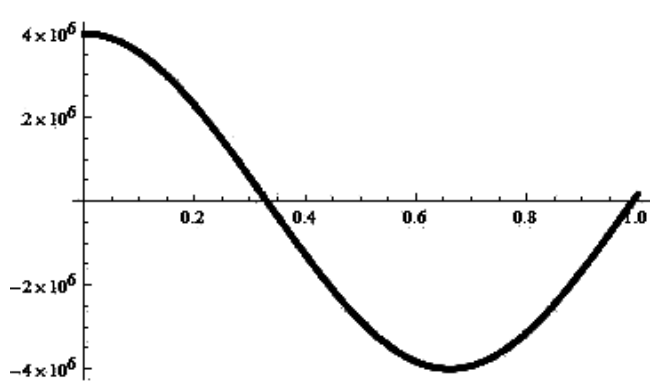

(a)

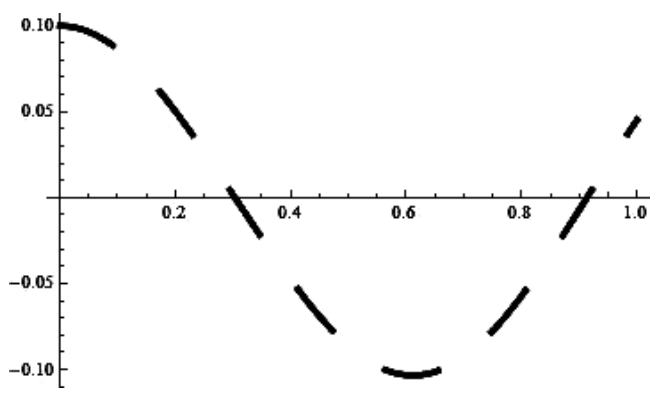

(b)

Fig. 6.2. 2-Type solution of problem (6.4): (a) the solution of problem (6.4) given for the initial data $\varepsilon_{2}^{\prime}(0)=0, \varepsilon_{2}(0)=4000000,(b)$ the difference between the neighboring solution and the solution $\varepsilon_{2}(t)$ of problem (6.4).

where $G$ is Green's function for the problem

$$
x^{\prime \prime}+\frac{\pi^{2}}{2} x=0, \quad x^{\prime}(0)=0, \quad x(1)=0 .
$$

It follows from (6.8) that

$$
|x(t)| \leq \Gamma \cdot M \quad \forall t \in[0,1]
$$

where $\Gamma=\frac{\sqrt{2}}{\pi \cos \frac{\pi}{\sqrt{2}}}$, holds.

Then

$$
x(t) \leq N \quad \forall t \in[0,1]
$$

satisfies, since

$$
\Gamma \cdot M=\frac{\sqrt{2}}{\pi \cos \frac{\pi}{\sqrt{2}}} \frac{3125}{191102976} \pi^{12} \approx 11.233<N \approx 117.828
$$

This means that problem (6.1) allows for quasilinearization for $k_{0}=\frac{\pi}{2}$ from the first nonresonance interval $(0 ; \pi \sqrt{2})$, and the solution $x(t)$ of the quasilinear BVP $(6.7),(6.2)$ is also a solution of a original resonant BVP (6.1), (6.2). From the Main Theorem 3.1 the follows that it is 1-type solution Fig. 6.1. Problem (6.1), (6.2) has also a 2-type solution Fig. 6.2. 
1. Agarwal R. P., O’Regan D. Ordinary and partial differential equations. - New York: Springer, 2009.

2. Conti R. Equazioni differenziali ordinarie quasilineari con condizioni lineari // Ann. mat. pura ed appl. 1962. - 57. - P. $49-67$.

3. Dobkevich $M$. On non-monotone approximation schemes for solutions of the second order differential equations // Different. and Integral Equat. - 2013. - 26, № 9-10, - P. 1169-1178.

4. Elsgolc L. E. Differential equations and calculus of variations. - Moscow: Nauka, 1969 (in Russian).

5. Tricomi F. G. Differential equations. - London: Blackie, 1961.

6. Yermachenko I., Sadyrbaev F. Types of solutions and multiplicity results for two-point nonlinear boundary value problems // Nonlinear Anal. - 2005. - 63. - P. e1725-e1735.

7. Yermachenko I., Sadyrbaev F. Quasilinearization and multiple solutions of the Emden-Fowler type equation // Math. Modelling and Anal. - 2005. - 10, № 1. - P. 41 -50.

Received 24.09.13, after revision - 03.01.14 